Tạp chí Khoa học và Công nghệ biển T10 (2010). Số 1. Tr 01 - 15

\title{
TƯƠNG TÁC GIŨ๋A VỎ VÀ NỀN SAN HÔ DƯớI TÁC DỤNG CỦA TẢI TRỌNG NỔ
}

\author{
NGUYẼ̃N THÁI CHUNG
}

\author{
Học viện Kỹ thuật Quân sự
}

\begin{abstract}
Tóm tắt. Bài báo trình bày phương pháp tính toán và một số kêt quả nghiên cúu đối với bài toán tương tác giữa kết cấu vỏ trụ kín và nền san hô chịu tác dụng của tải trọng động duới dạng sóng nổ. Bằng phwơng pháp phần tư hưu hạn (PTHH), bài toán đuợc giải quyết trên co sở kết hơp phần tử vỏ, phần tư khối và phần tủ tiếp xúc ba chiều (3D). Phần tư tiếp xúc được sủ dụng mô tả lớp tiếp xúc giữa vỏ và nền san hô đã thể hiện tính chất liên kết một chiều của nền san hô (chỉ chịu nén, không chịu kéo), do vậy, mô hình tính sát thực hơn sụ làm việc của kết cấu trong nền san hô. Nội dung của bài báo có thể làm tài liệu tham khảo cho việc nghiên cứu và tính toán, thiết kế các kết cấu công trình dạng vỏ trong nền san hô.
\end{abstract}

\section{MỞ ĐÀ̀U}

Đến nay việc nghiên cứu tính chất cơ lý của vật liệu san hô và nền san hô, cũng như nghiên cứu về địa chất công trình của nền san hô quần đảo Trường Sa phục vụ tính toán, thiết kế các công trình đã được tập trung quan tâm nghiên cứu và đã đạt được các kết quả đáng kể $[1,2]$, các kết quả đó là số liệu đầu vào cho việc tính toán, thiết kế các công trình biển, đảo nói chung. Các kết quả tính toán tương tác giữa kết cấu và nền san hô đến nay nhìn chung rất ít được công bố, kể cả trên Thế giới và trong nước, điều này có thể do thiếu số liệu đầu vào của nền san hô hoặc do tính chất bảo mật của từng Quốc gia. Nên việc tính toán, thiết kế và thi công các công trình trên nền san hô còn gặp rất nhiều khó khăn và hiệu quả kinh tế, an ninh Quốc phòng chưa cao. Nghiên cứu, đề xuất mô hình, phương pháp giải và tính toán cụ thể cho từng loại kết cấu công trình nhằm nâng cao hiệu quả kinh tế, sử dụng, phục vụ tốt cho an ninh Quốc phòng và kinh tế quốc dân là vấn đề cần thiết và có tính thời sự trong điều kiện hiện nay. Trong báo cáo này, tác giả sử dụng các kết quả nghiên cứu tính chất cơ lý về san hô và nền san hô $[1,2]$ làm số liệu đầu vào cho việc xây dựng mô hình, giải bài toán tương tác giữa kết cấu công trình dạng vỏ và nền san hô dưới tác dụng của tải trọng nổ trên cơ sở sử dụng kết hợp phần tử vỏ mỏng 4 điểm nút (mô tả kết cấu vỏ), phần tử lục diện 8 điểm nút (mô tả nền) và phần tử tiếp xúc ba chiều dạng lục diện 8 điểm nút (3D slip element) bằng phương pháp phần tử hữu hạn (PTHH) - đây là dạng kết cấu thường gặp trong các công trình quân sự trên các đảo san hô. 


\section{CÁC GIẢ THIẾT, MÔ HÌNH VÀ PHƯƠNG PHÁP TÍNH}

\section{Các giả thiết và mô hình tính của bài toán}

Bài toán được giải quyết dựa vào các giả thiết sau:

- Kết cấu vỏ đàn hồi, biến dạng tuyến tính.

- Mỗi lớp nền là vật liệu đồng nhất, đẳng hướng, đàn hồi tuyến tính. Quá trình kết cấu vỏ làm việc trong nền san hô, không có hiện tượng tách, trượt giữa các lớp nền với nhau.

- Hệ kết cấu vỏ và nền làm việc trong điều kiện biến dạng khối. Liên kết giữa kết cấu vỏ và nền san hô được thay thế bằng liên kết nút giữa các phần tử khối 3D thông qua liên kết với phần tử tiếp xúc (liên kết giữa phần tử thuộc kết cấu vỏ và phần tử thuộc nền thông qua phần tử trung gian - phần tử tiếp xúc $3 \mathrm{D}$ ). Liên kết tiếp xúc giữa kết cấu vỏ và nền san hô là liên kết một chiều.

• Khi tính toán, đối với vật liệu san hô, bỏ qua lực dính giữa kết cấu vỏ và nền.

Theo hướng này, tách từ hệ thực bán vô hạn ra một miền hữu hạn bao gồm kết cấu vỏ và một phần nền gọi là miền nghiên cứu, trên biên miền nghiên cứu được đặt các liên kết, việc tính toán được thực hiện trên miền nghiên cứu đã xác định. Biên của miền nghiên cứu được xác định dựa vào việc tính lặp theo thuật giải như sau $[1,5]$ :

Trước hết, chọn kích thước ban đầu bề rộng $\mathrm{B}_{0}$, chiều cao $\mathrm{H}_{0}$, chiều dài $\mathrm{L}_{0}$ :

Bước 1: Tính ứng suất lớn nhất $\sigma_{\max }^{(i)}$ tại biên nền khảo sát và trong vùng khảo sát.

Bước 2: Lập tỷ số đánh giá $\Delta \varepsilon_{\sigma_{\max }}=\frac{\sigma_{\max }-\sigma_{\max }^{(i)}}{\sigma_{\max }}$, với $\sigma_{\max }$ là ứng suất lớn nhất của nền trong vùng khảo sát.

Bước 3: Nếu $\Delta \varepsilon_{\sigma_{\max }} \leq \varepsilon$ (\#) thì $\mathrm{B}_{0}, \mathrm{H}_{0}$ và $\mathrm{L}_{0}$ là giá trị chấp nhận để tính toán. Ngược lại, chọn $\mathrm{B}_{1}=\mathrm{B}_{0}+\Delta \mathrm{B}_{0}, \mathrm{H}_{1}=\mathrm{H}_{0}+\Delta \mathrm{H}_{0}, \mathrm{~L}_{1}=\mathrm{L}_{0}+\Delta \mathrm{L}_{0}$ và tiên hành lặp lại từ bước 1. Quá trình lặp được thực hiện cho đến khi điều kiện (\#) được thoả mãn.

\section{Phương pháp tính và các loại PTHH}

Để tính toán kết cấu theo mô hình nói trên, tác giả sử dụng phương pháp PTHH, trong đó các loại phần tử sử dụng là: Đối với nền san hô sử dụng loại phần tử lục diện 8 nút đẳng tham số. Đối với vỏ là loại phần tử vỏ 4 nút. Đối với lớp tiếp xúc giữa kết cấu vỏ và nền san hô sử dụng loại phần tử tiếp xúc lục diện 8 điểm nút (solid slip element). 


\section{THIẾT LậP CÁC PHƯƠNG TRÌNH CƠ BẢN BẦNG PHƯỚNG PHÁP PTHH}

Với phần tử thuộc nền và vỏ là các loại phần tử được trình bày khá chi tiết trong các tài liệu PTHH $[3,4]$, cho nên trong phần này tác giả chỉ trình bày kỹ đối với loại phần tử tiếp xúc mô tả lớp tiếp xúc giữa vỏ và nền.

\section{Các quan hệ đối với phần tử khối thuộc nền}

Đối với các lớp nền san hô sử dụng phần tử khối lục diện 8 điểm nút đẳng tham số, tại mỗi nút có 3 bậc tự do là các chuyển vị nút theo các phương $\mathrm{x}, \mathrm{y}$ và $\mathrm{z}$ của hệ trục toạ độ tổng thể (hình 1). Các PTHH ở đây là các phần tử đẳng tham số, do đó cả dạng hình học và cả các hàm chuyển vị của nó là các tổ hợp tuyến tính của 8 hàm dạng như sau $[3,4]$ :

$$
\mathrm{N}_{\mathrm{i}}=\frac{1}{8}\left(1+\mathrm{rr}_{\mathrm{i}}\right)\left(1+\mathrm{ss}_{\mathrm{i}}\right)\left(1+\mathrm{tt}_{\mathrm{i}}\right) \text { với } \mathrm{i}=1,2,3,4, \ldots, 7,8
$$

trong đó: $\mathrm{r}, \mathrm{s}, \mathrm{t}$ là các toạ độ cục bộ, $\mathrm{r}_{\mathrm{i}}, \mathrm{s}_{\mathrm{i}}, \mathrm{t}_{\mathrm{i}}$ là các giá trị toạ độ cục bộ nút i (từ -1 đến 1 ).

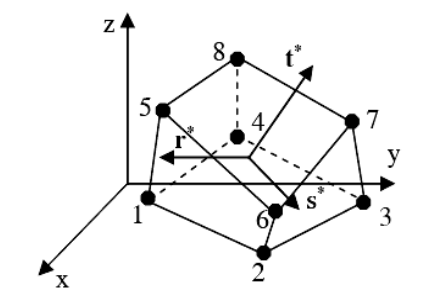

a, Trong hệ toạ độ chung

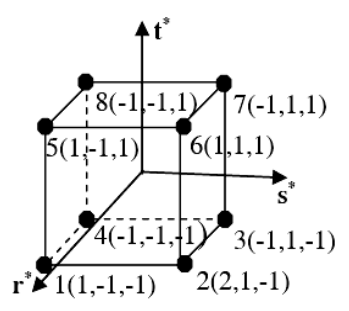

b, Trong hệ toạ độ tụ nhiên (cuc bộ)

Hình 1: Phần tử lục diện 8 điểm nút

Quan hệ giữa biến dạng $\{\varepsilon\}_{\mathrm{e}}$ tại 1 điểm trong phần tử và chuyển vị nút phần tử $\{\mathrm{q}\}_{\mathrm{e}}$ :

$$
\{\varepsilon\}_{\mathrm{e}}=[\mathrm{B}]\{\mathrm{q}\}_{\mathrm{e}},
$$

trong đó:

$$
\{\varepsilon\}_{\mathrm{e}}=\left\{\begin{array}{llllll}
\varepsilon_{\mathrm{x}} & \varepsilon_{\mathrm{y}} & \varepsilon_{\mathrm{z}} & \gamma_{\mathrm{xy}} & \gamma_{\mathrm{yz}} & \gamma_{\mathrm{zx}}
\end{array}\right\}^{\mathrm{T}},
$$

Ma trận độ cứng phần tử được xác định bởi: $[\mathrm{K}]_{\mathrm{e}}=\int_{\mathrm{V}_{\mathrm{e}}}[\mathrm{B}]^{\mathrm{T}}[\mathrm{D}][\mathrm{B}] \mathrm{dV}$,

trong đó $[\mathrm{B}]$ là ma trận vi phân hàm dạng, [D] là ma trận vật liệu.

Sử dụng phương pháp cầu phương Gauss với sơ đồ hai điểm Gauss, (4) trở thành: 


$$
\left[\mathrm{K}_{\mathrm{ij}}\right]=\sum_{\mathrm{i}=1}^{2} \sum_{\mathrm{j}=1 \mathrm{k}=1}^{2} \sum_{\mathrm{k}=1}^{2}\left(\left[\mathrm{~B}_{\mathrm{i}}\right]^{\mathrm{T}}[\mathrm{D}]\left[\mathrm{B}_{\mathrm{j}}\right]\right)_{\left(\mathrm{r}_{\mathrm{i}, \mathrm{j}}, \mathrm{t}_{\mathrm{k}}\right)},
$$

trong đó: $\left(\left[\mathrm{B}_{\mathrm{i}}\right]^{\mathrm{T}}[\mathrm{D}]\left[\mathrm{B}_{\mathrm{j}}\right]\right)_{\left(\mathrm{r}_{\mathrm{i}}, \mathrm{s}_{\mathrm{j}}, \mathrm{t}_{\mathrm{k}}\right)}$ là giá trị của $\left.\left(\left[\mathrm{B}_{\mathrm{i}}\right]^{\mathrm{T}}[\mathrm{D}]\left[\mathrm{B}_{\mathrm{j}}\right] \mathrm{J}\right)\right)$ tại các điểm tính Gauss có toạ độ $\left(\mathrm{r}_{\mathrm{i}}, \mathrm{s}_{\mathrm{j}}, \mathrm{t}_{\mathrm{k}}\right)$ với $\mathrm{r}_{\mathrm{i}}= \pm 0,57735 \ldots, \mathrm{s}_{\mathrm{j}}= \pm 0,57735 \ldots, \mathrm{t}_{\mathrm{k}}= \pm 0,57735$.

Véc tơ tải trọng nút do lực khối:

$$
\{P\}_{e}=\int_{V_{\mathrm{e}}}[\mathrm{N}]^{\mathrm{T}}\{\mathrm{g}\} \mathrm{dV},\{\mathrm{g}\}=\left\{\begin{array}{lll}
\mathrm{g}_{\mathrm{x}} & \mathrm{g}_{\mathrm{y}} & \mathrm{g}_{\mathrm{z}}
\end{array}\right\}
$$

Tại nút i, véc tơ lực khối được xác định:

$$
\left\{\mathrm{P}_{\mathrm{i}}\right\}=\sum_{\mathrm{i}=1}^{2} \sum_{\mathrm{j}=1 \mathrm{k}=1}^{2} \sum_{\mathrm{k}=1}^{2}\left\{\begin{array}{c}
\mathrm{N}_{\mathrm{i}} \mathrm{g}_{\mathrm{x}} \\
\mathrm{N}_{\mathrm{i}} \mathrm{g}_{\mathrm{y}} \\
\mathrm{N}_{\mathrm{i}} \mathrm{g}_{\mathrm{z}}
\end{array}\right]|\mathrm{J}|_{\left(\mathrm{F}_{\mathrm{i}}, \mathrm{s}_{\mathrm{j}, \mathrm{t}} \mathrm{\textrm {k } _ { \mathrm { k } }}\right)}
$$

với: $\left[\mathrm{N}_{\mathrm{i}}\right]=\left[\begin{array}{ccc}\mathrm{N}_{\mathrm{i}} & 0 & 0 \\ 0 & \mathrm{~N}_{\mathrm{i}} & 0 \\ 0 & 0 & \mathrm{~N}_{\mathrm{i}}\end{array}\right],\{\mathrm{g}\}=\left\{\begin{array}{l}\mathrm{g}_{\mathrm{x}} \\ \mathrm{g}_{\mathrm{y}} \\ \mathrm{g}_{\mathrm{z}}\end{array}\right\}$ và $|\mathrm{J}|$ là định thức ma trận Jacobin.

\section{Các quan hệ đối với phần tử khối thuộc lớp tiếp xúc (PTTX)}

Mô hình hình học của phần tử tiếp xúc 3 chiều (3D) được biểu diễn dưới dạng hình lục diện 8 nút với hệ trục toạ độ cục bộ và tổng thể của nó như trên hình $2[6,7,8]$. Tại mỗi nút của phần tử tiếp xúc có 3 bậc tự do, thành phần chuyển vị là hàm của các toạ độ. Nhằm đảm bảo sự tương thích với các phần tử vỏ và phần tử khối của lớp nền, các cặp nút đối diện mặt trên và dưới của phần tử tương ứng là 1 và 5,2 và 6,3 và 7,4 và 8 có cùng toạ độ, tức là thực tế phần tử có "độ mở” bằng không.

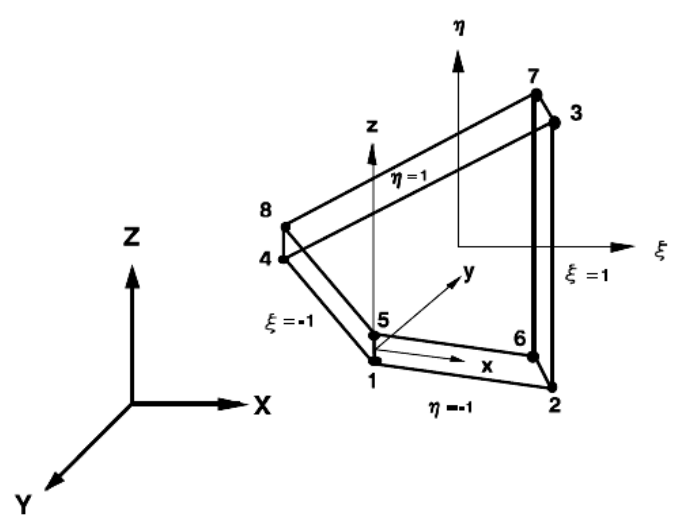

Hình 2: Phần tử tiếp xúc 3 chiều 
Ma trận độ cứng $\left[K^{\prime}\right]$ của phần tử tiếp xúc trong hệ toạ độ cục bộ:

$\left[\mathrm{K}^{\prime}\right]=\iint[\mathrm{N}]^{\mathrm{T}}[\mathrm{k}][\mathrm{N}] \mathrm{dxdy}$,

trong đó $[\mathrm{k}]$ là ma trận bao gồm độ cứng đàn hồi theo phương tiếp tuyến và phương pháp tuyến của phần tử, được xác định theo công thức:

$$
[\mathrm{k}]=\left[\begin{array}{ccc}
\mathrm{k}_{\mathrm{sx}} & 0 & 0 \\
0 & \mathrm{k}_{\mathrm{sy}} & 0 \\
0 & 0 & \mathrm{k}_{\mathrm{nz}}
\end{array}\right],
$$

với: $\mathrm{k}_{\mathrm{sx}}$ và $\mathrm{k}_{\mathrm{sy}}$ là độ cứng tiếp tuyến theo phương $\mathrm{x}$ và phương $\mathrm{y}$ (nếu vật liệu đẳng hưởng, ta có: $\mathrm{k}_{\mathrm{sx}}=\mathrm{k}_{\mathrm{sy}}$ ), $\mathrm{k}_{\mathrm{nz}}$ là độ cứng pháp tuyến theo phương $\mathrm{z}$. Đặc trưng của phần tử tiếp xúc thể hiện trong bảng 1 .

Quan hệ số gia ứng suất và số gia biến dạng trong phần tử tiếp xúc được đặc trưng bởi phương trình:

$$
\left\{\begin{array}{l}
\Delta \sigma \\
\Delta \tau_{\mathrm{zx}} \\
\Delta \tau_{\mathrm{zy}}
\end{array}\right\}=[\mathrm{k}]\left\{\begin{array}{l}
\Delta \varepsilon_{\mathrm{z}} \\
\Delta \gamma_{\mathrm{zx}} \\
\Delta \gamma_{\mathrm{zy}}
\end{array}\right\},
$$

trong đó: $\Delta \sigma, \Delta \varepsilon_{\mathrm{z}}$ tương ứng là số gia ứng suất và số gia biến dạng theo phương pháp tuyến $\mathrm{z}, \Delta \tau_{\mathrm{zx}}, \Delta \gamma_{\mathrm{zx}}$ tương ứng là số gia ứng suất và số gia biến dạng theo phương tiếp tuyến $\mathrm{x}, \Delta \tau_{\mathrm{zy}}, \Delta \gamma_{\mathrm{zy}}$ tương ứng là số gia ứng suất và số gia biến dạng theo phương tiếp tuyến $\mathrm{y}$.

Quan hệ giữa số gia chuyển vị nút phần tử trong hệ toạ độ chung xoy với các số gia biến dạng pháp tuyến và biến dạng tiếp tuyến được xác định theo công thức sau:

$$
\left\{\begin{array}{l}
\Delta \varepsilon \\
\Delta \gamma_{\mathrm{zx}} \\
\Delta \gamma_{\mathrm{zy}}
\end{array}\right\}=[\mathrm{N}]\left\{\Delta \mathrm{U}_{\mathrm{se}}\right\},
$$

trong đó: $\left\{\Delta \mathrm{U}_{\mathrm{se}}\right\}$ là véc tơ số gia chuyển vị nút của phần tử tiếp xúc trong hệ toạ độ chung, $\left\{\Delta \mathrm{U}_{\mathrm{se}}\right\}=\left\{\begin{array}{lllllll}\delta \mathrm{u}_{1} & \delta \mathrm{v}_{1} & \delta \mathrm{w}_{1} & \ldots & \delta \mathrm{u}_{8} & \delta \mathrm{v}_{8} & \delta \mathrm{w}_{8}\end{array}\right\}^{\mathrm{T}}$.

Trên hình 3 thể hiện quan hệ giữa ứng suất và biến dạng trong phần tử. 

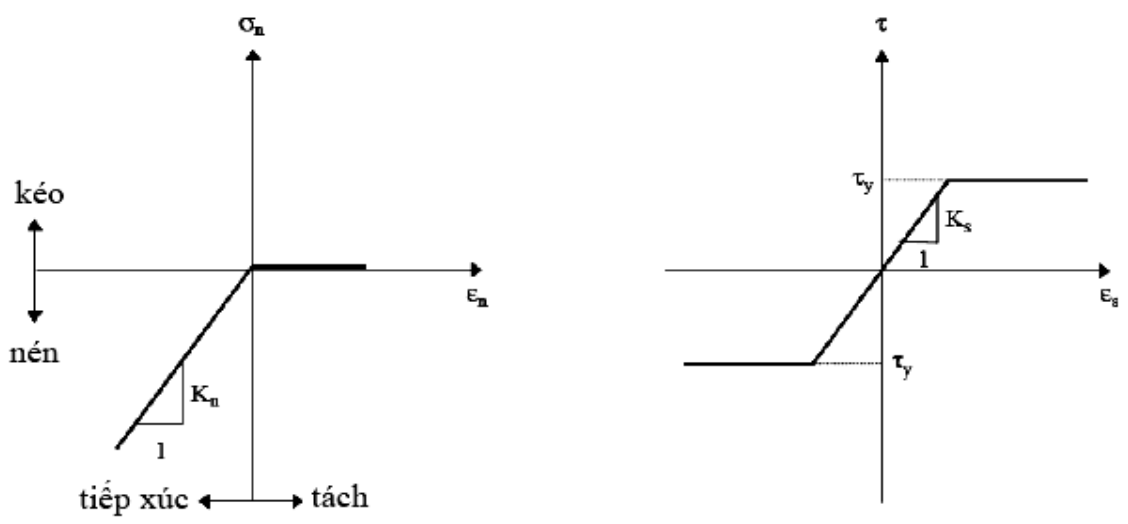

a, Quan hệ ứng suất - biến dạng pháp tuyến; b, Quan hệ ưng suất - biến dạng tiếp tuyến

Hình 3: Quan hệ giữa ứng suất và biến dạng trong phần tử tiếp xúc

Thay trong (7) các biến tích phân bởi:

$$
\mathrm{dxdy}=\operatorname{Det}[\mathrm{J}] \mathrm{d} \xi \mathrm{d} \eta,
$$

trong đó $[\mathrm{J}]$ là ma trận Jacobi. Ma trận độ cứng lúc này có thể được tính toán bằng phương pháp tích phân số với việc sử dụng công thức cầu phương Gauss. Thông thường, với $\mathrm{n}$ điểm tích phân Gauss, có thể viết:

$$
\left[K^{\prime}\right]=\sum_{i=1}^{n} \sum_{j=1}^{n}\left[N\left(\xi_{i}, \eta_{i}\right)\right]^{T}[k]\left[N\left(\xi_{i}, \eta_{i}\right)\right] \operatorname{det}[J] \mathrm{w}_{i} w_{j},
$$

trong đó: $\mathrm{w}_{\mathrm{i}}$ và $\mathrm{w}_{\mathrm{j}}$ là các trọng số.

Bảng 1: Đặc trưng vật liệu của phần tử tiếp xúc (vật liệu đẳng hướng)

\begin{tabular}{|c|c|c|c|c|}
\hline TT & Đặc trưng vật liệu & Ký hiệu & Thứ nguyên & Cách tính \\
\hline 1 & Lực dính đơn vị & $\mathrm{C}$ & Lực/(ch.dài) ${ }^{2}$ & Cho trước \\
\hline 2 & Góc ma sát trong & $\varphi$ & độ & Cho trước \\
\hline 3 & Độ cứng pháp tuyến & $\mathrm{k}_{\mathrm{nz}}$ & Lực/(ch.dài) $)^{2}$ & $\mathrm{k}_{\mathrm{nz}}=\frac{\mathrm{E}(1-v)}{(1+v)(1-2 v)}$ \\
\hline 4 & Độ cứng tiếp tuyến & $\mathrm{k}_{\mathrm{sx}}, \mathrm{k}_{\mathrm{sy}}$ & Lực/(ch.dài) & $\mathrm{k}_{\mathrm{sx}}=\mathrm{k}_{\mathrm{sy}}=\frac{\mathrm{E}}{2(1+\mathrm{v})}$ \\
\hline 5 & Độ cứng chống trượt tới hạn & $\mathrm{k}_{\mathrm{res}}$ & Lực/(ch.dài) & $\mathrm{k}_{\mathrm{res}}=\mathrm{G}_{\mathrm{res}}$ \\
\hline
\end{tabular}


Khi tính toán tương tác giữa vỏ và nền san hô, số liệu xác định đặc trưng của phần tử tiếp xúc được lấy là số liệu đặc trưng của nền. Khi ma trận độ cứng của phần tử tiếp xúc trong hệ trục toạ độ tổng thể được xác định thì các số hạng của ma trận này ghép nối vào ma trận độ cứng của hệ thống được tiến hành như với các phần tử hữu hạn khác bằng phương pháp cộng trực tiếp đã biết $[3,4]$, trong đó, các thành phần gây ra cùng một loại chuyển vị được góp phần vào vị trí tương ứng trên ma trận tổng thể.

\section{Các quan hệ đối với phần tử vỏ thuộc kết cấu vỏ}

Vỏ được rời rạc hoá bởi hữu hạn các phần tử vỏ dạng tứ giác 4 điểm nút, mỗi nút có 6 bậc tự do: $\left\{\mathrm{q}_{\mathrm{i}}\right\}=\left\{\begin{array}{llllll}\mathrm{u}_{\mathrm{i}} & \mathrm{v}_{\mathrm{i}} & \mathrm{w}_{\mathrm{i}} & \theta_{\mathrm{xi}} & \theta_{\mathrm{yi}} & \theta_{\mathrm{zi}}\end{array}\right\}, \mathrm{i}=1 \div 4$, trong đó $\mathrm{u}_{\mathrm{i}}, \mathrm{v}_{\mathrm{i}}$ là chuyển vị nút trong mặt phẳng trung bình vỏ (trạng thái ứng suất phẳng), $\mathrm{w}_{\mathrm{i}}, \theta_{\mathrm{xi}}, \theta_{\mathrm{yi}}$ là chuyển vị các nút trong trạng thái chịu uốn, các thành phần độ cứng tương ứng của chúng được xác định theo phần tử tấm chịu uốn và kéo (nén) thông thường $[3,4]$. Đại lượng $\theta_{\mathrm{zi}}$ là góc xoay quanh trục $\mathrm{z}$ của hệ trục toạ độ cục bộ phần tử, các thành phần độ cứng ứng với bậc tự do này nhận được qua phép biến đổi ma trận phần tử $\left[\mathrm{K}^{\mathrm{e}}\right]$ từ hệ trục cục bộ sang hệ toạ độ chung theo công thức: $[\mathrm{K}]=[\mathrm{T}]^{\mathrm{T}}\left[\mathrm{K}^{\mathrm{e}}\right][\mathrm{T}]$, với $[\mathrm{T}]$ là ma trận chuyển toạ độ. Ma trận độ cứng $\left[\mathrm{K}^{\mathrm{e}}\right]$ và ma trận khối lượng $\left[\mathrm{M}^{\mathrm{e}}\right]$ của phần tử có cấp $24 \times 24$ được xác định theo [3].

\section{Phương trình chuyển động của kết cấu}

Dưới tác dụng của tải trọng động phương trình chuyển động của hệ như sau [1, 2, 3]:

$$
[\mathrm{M}]\{\ddot{\mathrm{U}}\}+[\mathrm{C}]\{\dot{\mathrm{U}}\}+[\mathrm{K}]\{\mathrm{U}\}=\{\mathrm{R}\},
$$

trong đó: $[\mathrm{M}]=\sum_{\mathrm{e}}[\mathrm{M}]_{\mathrm{e}}$ là ma trận khối lượng tổng thể của hệ, $[\mathrm{C}]=\alpha[\mathrm{M}]+\beta[\mathrm{K}]$ là ma trận cản tổng thể: $\beta=\frac{2 \xi}{\omega_{1}+\omega_{2}} ; \alpha=\beta \omega_{1} \omega_{2} ;\left(\omega_{1}, \omega_{2}\right.$ là 2 tần số đầu tiên trong dao động tự do, $\xi$ là tỷ số cản), $[K]=\sum_{\mathrm{e}}[\mathrm{K}]_{\mathrm{e}}$ là ma trận độ cứng tổng thể của hệ và $\{\mathrm{R}\}$ là véc tơ ngoại lực nút của hệ, bao gồm tải trọng ngoài, ứng suất ban đầu, biến dạng ban đầu.

Ghép nối các ma trận phần tử vỏ và ma trận phần tử khối (bao gồm nền và lớp tiếp xúc) để tạo thành ma trận tổng thể của kết cấu bằng phương pháp độ cứng trực tiếp sẽ sử dụng mảng lưu trữ địa chỉ nút và sơ đồ Skyline $[2,3]$, trong đó, đối với các nút chung giữa phần tử vỏ và phần tử khối thì số hạng trong ma trận số độ cứng (ma trận khối lượng) phần tử khối được cộng vào số hạng có cùng bậc tự do của phần tử vỏ chung nút theo quy tắc ma trận chỉ số. Xét đến sự tách, trượt giữa vỏ và nền, các ma trận cản, ma trận độ cứng tổng thể của hệ phụ thuộc chuyển vị nút $\{U\}$, nên (13) là phương trình phi tuyến hình học. 


\section{THUẬT TOÁN GIẢI PHƯƠNG TRÌNH CHUYỂN ĐỘNG CỦA KẾT CÁU}

Phương trình (13) khi tính đến sự trượt và tách trên bề mặt tiếp xúc giữa vỏ và nền sẽ có dạng phi tuyến như đã nói ở trên và được viết lại dưới dạng $[4,5]$ :

$$
[\mathrm{M}]\{\ddot{\mathrm{U}}\}+[\mathrm{C}\{\mathrm{U}\}]\{\dot{\mathrm{U}}\}+[\mathrm{K}\{\mathrm{U}\}\}\{\mathrm{U}\}=\{\mathrm{R}\} .
$$

Để giải phương trình chuyển động dạng phi tuyến (14) sẽ sử dụng phương pháp tích phân trực tiếp Newmark kết hợp với phương pháp lặp Newton - Raphson, theo đó nghiệm phương trình (14) tại bước lặp thứ i của thời điểm tính $\mathrm{t}+\Delta \mathrm{t}$ xác định bởi hệ phương trình:

$$
\begin{aligned}
& \left.[\mathrm{M}]\left\{\ddot{\mathrm{U}}_{\mathrm{t}+\Delta t}\right\}\right\}^{(\mathrm{i})}+\left[\mathrm{C}_{\mathrm{t}+\Delta t}\{\mathrm{U}\}\right]^{(\mathrm{i}-1)}\left\{\dot{\mathrm{U}}_{\mathrm{t}+\Delta t}\right\}^{(\mathrm{i})}+\left[\mathrm{K}_{\mathrm{t}+\Delta t}\{\mathrm{U}\}\right]^{(\mathrm{i}-1)}\{\Delta \mathrm{U}\}^{(\mathrm{i})}= \\
& \left\{\mathrm{R}_{\mathrm{t}+\Delta t}\right\}-\left\{\mathrm{P}_{\mathrm{t}+\Delta t}\right\}^{\left(\mathrm{i}^{i}\right)}, \\
& \left\{\mathrm{U}_{\mathrm{t}+\Delta t}\right\}^{(\mathrm{i})}=\left\{\mathrm{U}_{\mathrm{t}+\Delta t}\right\}^{(\mathrm{i}-1)}+\{\Delta \mathrm{U}\}^{(\mathrm{i})},
\end{aligned}
$$

với: $\left\{\mathrm{P}_{\mathrm{t}+\Delta t}\right\}^{(\mathrm{i})}$ là véc tơ nội lực quy nút của hệ tại bước lặp $\mathrm{i}$ (chỉ số $\mathrm{i}$ chỉ thứ tự bước lặp), $\{\Delta \mathrm{U}\}^{(\mathrm{i})}$ là véc tơ số gia chuyển vị nút tại bước lặp thứ i

Vận tốc và gia tốc tại thời điểm $\mathrm{t}+\Delta \mathrm{t}$ của bước lặp thứ i là:

$$
\begin{aligned}
& \left\{\dot{U}_{\mathrm{t}+\Delta t}\right\}^{(i)}=a_{1}\left(\left\{\mathrm{U}_{\mathrm{t}+\Delta t}\right\}^{(i-1)}+\{\Delta \mathrm{U}\}^{(\mathrm{i})}-\left\{\mathrm{U}_{\mathrm{t}}\right\}\right)-\mathrm{a}_{4}\left\{\dot{U}_{\mathrm{t}}\right\}-\mathrm{a}_{5}\left\{\ddot{U}_{\mathrm{t}}\right\}, \\
& \left\{\ddot{\mathrm{U}}_{\mathrm{t}+\Delta t}\right\}^{(\mathrm{i})}=\mathrm{a}_{0}\left(\left\{\mathrm{U}_{\mathrm{t}+\Delta t}\right\}^{(\mathrm{i}-1)}+\{\Delta \mathrm{U}\}^{(\mathrm{i})}-\left\{\mathrm{U}_{\mathrm{t}}\right\}\right)-\mathrm{a}_{2}\left\{\dot{U}_{\mathrm{t}}\right\}-\mathrm{a}_{3}\left\{\ddot{\mathrm{U}}_{\mathrm{t}}\right\},
\end{aligned}
$$

trong đó:

$$
\begin{aligned}
& \mathrm{a}_{0}=\frac{1}{\alpha \Delta \mathrm{t}^{2}} ; \quad \mathrm{a}_{1}=\frac{\delta}{\alpha \Delta \mathrm{t}} ; \quad \mathrm{a}_{2}=\frac{1}{\alpha \Delta \mathrm{t}} ; \quad \mathrm{a}_{3}=\frac{1}{2 \alpha}-1 ; \\
& \mathrm{a}_{4}=\frac{\delta}{\alpha}-1 ; \quad \mathrm{a}_{5}=\frac{\Delta \mathrm{t}}{2}\left(\frac{\delta}{\alpha}-2\right) ;
\end{aligned}
$$

với $\delta$ và $\alpha$ là các tham số, được chọn với điều kiện: $\delta \geq 0,5 ; \alpha \geq 0,25(0,5+\delta)^{2}$.

Điều kiện ban đầu cho mỗi cấp tải trọng được xác định như sau:

$$
\left\{\mathrm{U}_{\mathrm{t}+\Delta \mathrm{t}}\right\}^{(0)}=\left\{\mathrm{U}_{\mathrm{t}}\right\}, \quad\left\{\mathrm{P}_{\mathrm{t}+\Delta \mathrm{t}}\right\}^{(0)}=\left\{\mathrm{P}_{\mathrm{t}}\right\}, \quad\left\{\mathrm{K}_{\mathrm{t}+\Delta \mathrm{t}}\right\}^{(0)}=\left\{\mathrm{K}_{\mathrm{t}}\right\}
$$

Sử dụng ma trận độ cứng tiếp tuyến hiệu quả $\left[\mathrm{K}_{\mathrm{t}+\Delta t}^{*}\right]^{(\mathrm{i}-1)}$, véc tơ tải trọng hiệu quả $\left\{\mathrm{R}_{\mathrm{t}+\Delta \mathrm{t}}^{*}\right\}$, véc tơ nội lực $\left\{\mathrm{P}_{\mathrm{t}+\Delta t}\right\}^{(\mathrm{i})}$, phương trình (15) được viết lại dưới dạng:

$$
\left[\mathrm{K}_{\mathrm{t}+\Delta t}^{*}\right]^{(\mathrm{i}-1)}\{\Delta \mathrm{U}\}^{(\mathrm{i})}=\left\{\mathrm{R}_{\mathrm{t}+\Delta \mathrm{t}}^{*}\right\}-\left\{\mathrm{P}_{\mathrm{t}+\Delta t}\right\}^{(\mathrm{i})} .
$$


Ma trận cản $\left[\mathrm{C}_{\mathrm{t}+\Delta t}\right]^{(\mathrm{i}-1)}$ ở bước lặp (i-1) tại thời điểm $\mathrm{t}+\Delta \mathrm{t}$ được tính theo tổ hợp tuyến tính của ma trận độ cứng và ma trận khối lượng tổng thể của hệ:

$$
\left[\mathrm{C}_{\mathrm{t}+\Delta \mathrm{t}}\right]^{(\mathrm{i}-1)}=\alpha[\mathrm{M}]+\beta\left[\mathrm{K}_{\mathrm{t}+\Delta \mathrm{t}}\right]^{(\mathrm{i}-1)} .
$$

Trong quá trình tính, kiểm tra điều kiện bền về tách và trượt trên bề mặt tiếp xúc của vỏ với nền đàn hồi được thực hiện theo tiêu chuẩn bền Morh - Coulumb $[6,7,8]$.

Tiêu chuẩn dừng của phép lặp là sự hội tụ của chuyển vị nút:

$$
\frac{\left\|\{\Delta \mathrm{U}\}^{(\mathrm{i})}\right\|}{\left\|\left\{\mathrm{U}_{\mathrm{t}+\Delta \mathrm{t}}\right\}^{(\mathrm{i})}-\left\{\mathrm{U}_{\mathrm{t}}\right\}\right\|} \leq \varepsilon_{\mathrm{D}}
$$

Để giải bài toán đã nêu, đối với mỗi cấp tải trọng (ứng với mỗi bước thời gian $\Delta \mathrm{t}$ ), tiến hành giải lặp bài toán phi tuyến liên kết, với thuật toán như sau:

1. Gán điều kiện ban đầu của phép lặp như (20).

2. Giải bài toán trị riêng xác định tần số, tính ma trận cản nhớt tổng thể theo (22).

3. Tính ma trận độ cứng hiệu quả $\left[\mathrm{K}_{\mathrm{t}+\Delta t}^{*}\right]^{(\mathrm{i}-1)}$.

4. Tính véc tơ tải trọng hiệu quả $\left\{\mathrm{R}_{\mathrm{t}+\Delta t}^{*}\right\}$.

5. Tính số gia chuyển vị $\{\Delta \mathrm{U}\}^{(\mathrm{i})}$ theo giá trị hiện thời của véc tơ tải trọng $\left\{\mathrm{R}_{\mathrm{t}+\Delta \mathrm{t}}\right\}$ khi giải hệ phương trình (21).

6. Tính luỹ tích của véc tơ chuyển vị nút $\left\{\mathrm{U}_{\mathrm{t}+\Delta \mathrm{t}}\right\}^{(\mathrm{i})}$ theo (16). Với mỗi phần tử tiếp xúc, thực hiện các bước từ 7 đến 14 :

7. Tính số gia biến dạng $\{\Delta \varepsilon\}^{(\mathrm{i})}$ của phần tử tiếp xúc theo $(10)$.

8. Tính số gia ứng suất $\left\{\Delta \sigma_{\mathrm{t}+\Delta t}^{\mathrm{se}}\right\}^{(\mathrm{i})}$ trong phần tử tiếp xúc tương ứng với số gia biến dạng $\{\Delta \varepsilon\}^{(\mathrm{i})}$ theo $(9)$.

9. Tính ứng suất của phần tử tiếp xúc: $\left\{\sigma_{\mathrm{t}+\Delta t}^{\mathrm{se}}\right\}^{(\mathrm{i})}=\left\{\sigma_{\mathrm{t}}^{\mathrm{se}}\right\}+\left\{\Delta \sigma_{\mathrm{t}+\Delta t}^{\mathrm{se}}\right\}^{(\mathrm{i})}$.

10. Tính ứng suất tiếp giới hạn trên mặt tiếp xúc theo tiêu chuẩn Morh - Coulomb: $\tau_{\mathrm{gh}}=\mathrm{C}+\sigma \operatorname{tg} \varphi$.

11. Kiểm tra sự tách, trượt trên mặt tiếp xúc ở bước 9 và 10 và cập nhật ma trận độ cứng của phần tử tiếp xúc: 


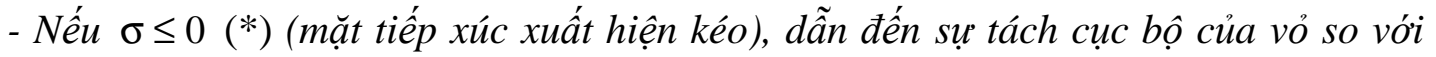
nền $\Rightarrow k_{n z}=0$ và $k_{s x}, k_{s y}=0$. Song để xét được quá trình chập lại của khe hở và kết quả tính không suy biến, khi lập trình ta tiến hành gán cho chúng giá trị $=10^{-4}$ giá trị ban đầu $\left(k_{n z}=10^{-4} k_{n z}, k_{s x}=10^{-4} k_{s x}, k_{s y}=10^{-4} k_{s y}\right.$ ) [8]. Tiến hành lặp lại tù bước 2.

- Nếu $\sigma>0$ và $\tau<\tau_{\mathrm{gh}}$ trên mặt tiếp không xảy ra truợt hay tách cục bộ của tấm so với lớp đệm, độ cưng $k_{n z}$ và $k_{s x}, k_{s y}$ được cập nhật theo bảng 1. Tiến hành lặp lại tù buớc 3.

- Nếu $\sigma>0$ và $\tau \geq \tau_{\mathrm{gh}}$ trên mặt tiếp xúc xảy ra truợt cục bộ của tấm so với lóp đệm nhung tồn tại lực ma sát không đổi, độ cứng $k_{s x}=k_{s y}=k_{\text {res, }}, k_{n z}$ được cập nhật theo bảng 1 . Tiến hành lặp lại tù̀ bước 2.

- Khi dõ tải, nếu $\sigma \leq 0$, tiếp tục sủ dụng giá trị $k_{n z}=10^{-4} k_{n z}, k_{s x}=10^{-4} k_{s x}, k_{s y}=10^{-}$ ${ }^{4} k_{s y}$. Nếu $\sigma>0$, ta cập nhật độ cứng $k_{n z}$ và $k_{s x}$, $k_{s y}$ theo bảng 1 .

12. Tính ma trận độ cứng $\left[\mathrm{K}^{\prime}\right]$ của phần tử tiếp xúc theo giá trị mới của $\mathrm{k}_{\mathrm{nz}}$ và $\mathrm{k}_{\mathrm{sx}}$, $\mathrm{k}_{\mathrm{sy}}$, cập nhật ma trận độ cứng tổng thể $\left[\mathrm{K}_{\mathrm{t}+\Delta \mathrm{t}}\right]^{(\mathrm{i})}$.

13. Tính nội lực nút của phần tử tiếp xúc theo ứng suất $\left\{\sigma_{t+\Delta t}^{\text {se }}\right\}^{(i)}$ của phần tử và cập nhật véc tơ nội lực $\left\{\mathrm{P}_{\mathrm{t}+\Delta t}\right\}^{(\mathrm{i})}$.

14. Kết thúc chu trình tính cho phần tử tiếp xúc.

15. Cập nhật ma trận độ cứng tổng thể $\left[\mathrm{K}_{\mathrm{t}+\Delta t}\right]^{(\mathrm{i})}$ từ các ma trận độ cứng của các phần tử vỏ, nền và phần tử tiếp xúc.

16. Tính véc tơ nội lực các phần tử vỏ và phần tử nền theo trạng thái ứng suất mới $\left\{\sigma_{t+\Delta t}\right\}^{(i)}$, cập nhật véc tơ nội lực $\left\{\mathrm{P}_{\mathrm{t}+\Delta t}\right\}^{(\mathrm{i})}$.

17. Kiểm tra điều kiện hội tụ (23) và sơ đồ tiếp xúc tại bước thứ (i) trùng với sơ đồ tiếp xúc tại bước thứ (i-1). Nếu không thoả mãn thì tính lặp tiếp từ bước 2 của thuật toán trên.

\section{VÍ DỤ Số}

Với các thuật toán nêu trên, tiến hành lập trình để giải bài toán trên máy tính. Chương trình có tên SHELL_Coral_Dynamic, được viết bằng ngôn ngữ Matlab, chạy trên máy tính với hệ điều hành Window xp.

Tính toán kết cấu vỏ trụ kín làm bằng vật liệu thép, dày $0.7 \mathrm{~mm}$, đường kính $30 \mathrm{~cm}$, chiều dài $80 \mathrm{~cm}$ nằm thẳng đứng trong nền san hô 4 lớp (bảng 2), chịu tác dụng của tải 
trọng nổ tác dụng theo phương ngang (nổ trong lòng nền). Tải trọng $\mathrm{p}(\mathrm{t})$ phân bố đều trên tiết diện nền tính toán, chấp nhận mô hình gần đúng, theo [2], ta có:

$$
\mathrm{p}(\mathrm{t})=\left\{\begin{array}{lll}
\mathrm{p}_{\max }\left(1-\frac{\mathrm{t}}{\tau}\right) & \text { khi } & 0<\mathrm{t}<\tau \\
0 & \text { khi } \mathrm{t} \geq \tau
\end{array}, \text { với: } \mathrm{p}_{\max }=\mathrm{A}\left(\frac{\sqrt[3]{\mathrm{C}}}{\mathrm{R}}\right)^{\mathrm{m}} \text {, trong đó: } \mathrm{A}\right. \text { và m là các }
$$

hằng số thực nghiệm, $\mathrm{C}$ là khối lượng thuốc nổ $\mathrm{TNT}$ tính bằng $(\mathrm{kg})$ và $\mathrm{R}$ là khoảng cách từ tâm nổ. Bằng thực nghiệm đã xác định được các hệ số: $\left\{\begin{array}{l}A=472.1393 \\ m=0.2107\end{array}\right.$. Trong bài toán đặt $\mathrm{ra}$, với bán kính mặt cắt xác định tải trọng $\mathrm{p}(\mathrm{t})$ là $\mathrm{R}=1 \mathrm{~m}$, ta có: $\mathrm{p}_{\max }=308.8128 \mathrm{~kg} / \mathrm{m}^{2}$, với thời gian duy trì tải trọng $\tau=0.05 \mathrm{~s}$ đồ thị hàm tải trọng được thể hiện như hình 4 .

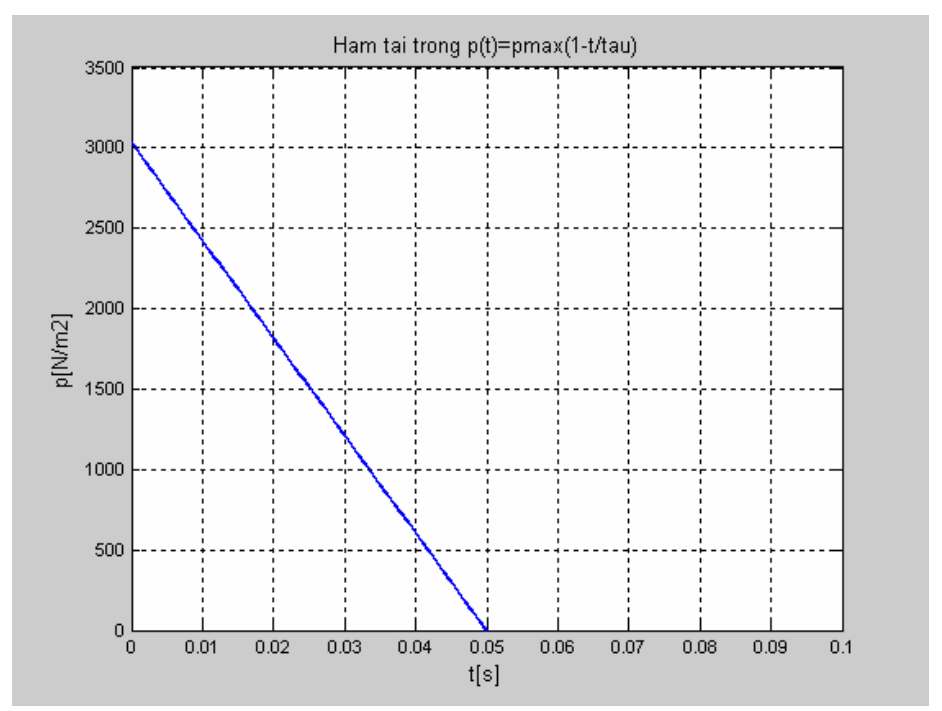

Hình 4: Hàm tải trọng

Bảng 2: Thuộc tính vật liệu [1]

\begin{tabular}{|c|c|c|c|c|c|c|}
\hline Lớp & $\begin{array}{c}\text { Độ sâu } \\
\text { (m) }\end{array}$ & $\begin{array}{c}E \\
\left(\mathrm{~N} / \mathrm{cm}^{2}\right)\end{array}$ & $v$ & $\begin{array}{c}\rho \\
\left(\mathrm{kg} / \mathrm{cm}^{3}\right)\end{array}$ & $\begin{array}{c}\text { Hệ số ma sát } \\
\text { f }\end{array}$ & $\begin{array}{c}\text { Hệ số giảm chấn } \\
\xi\end{array}$ \\
\hline 1 & 2 & $2.8 \times 10^{4}$ & $0 \times 22$ & $2.5 \times 10^{-3}$ & 0.21 & \multirow{4}{*}{0.05} \\
\hline 2 & 6 & $2.1 \times 10^{5}$ & $0 \times 25$ & $2.8 \times 10^{-3}$ & 0.32 & \\
\hline 3 & 10 & $2.0 \times 10^{6}$ & $0 \times 25$ & $2.9 \times 10^{-3}$ & 0.41 & \\
\hline 4 & 16 & $2.6 \times 10^{5}$ & $0 \times 25$ & $2.0 \times 10^{-3}$ & 0.47 & \\
\hline
\end{tabular}


Mô hình PTHH bài toán như hình 5.

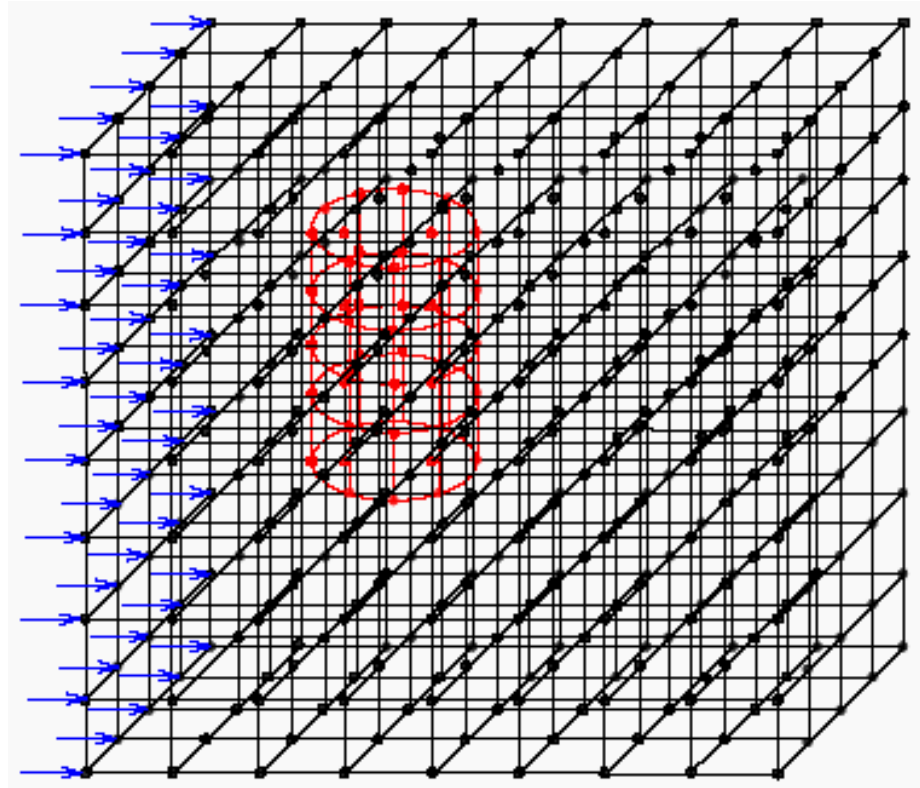

Hình 5: Mô hình PTHH của bài toán

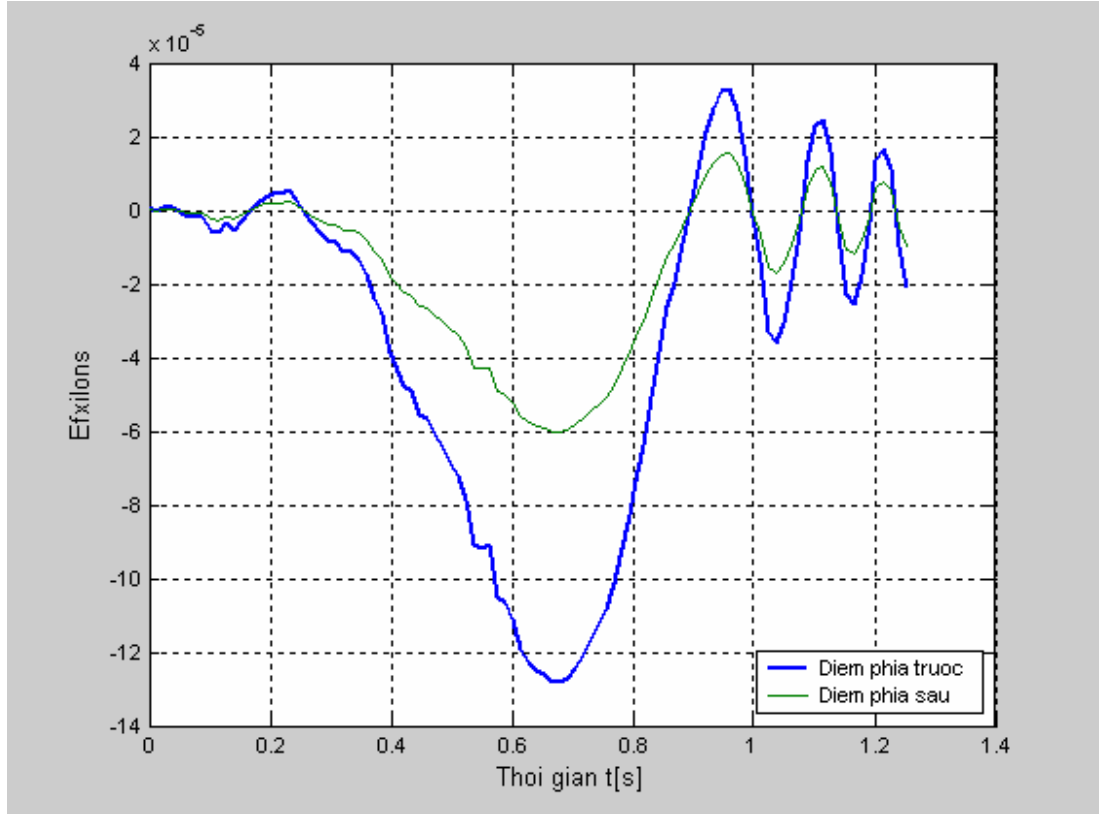

Hình 6: Biến dạng $\varepsilon_{\varphi}$ tại các điểm tính 


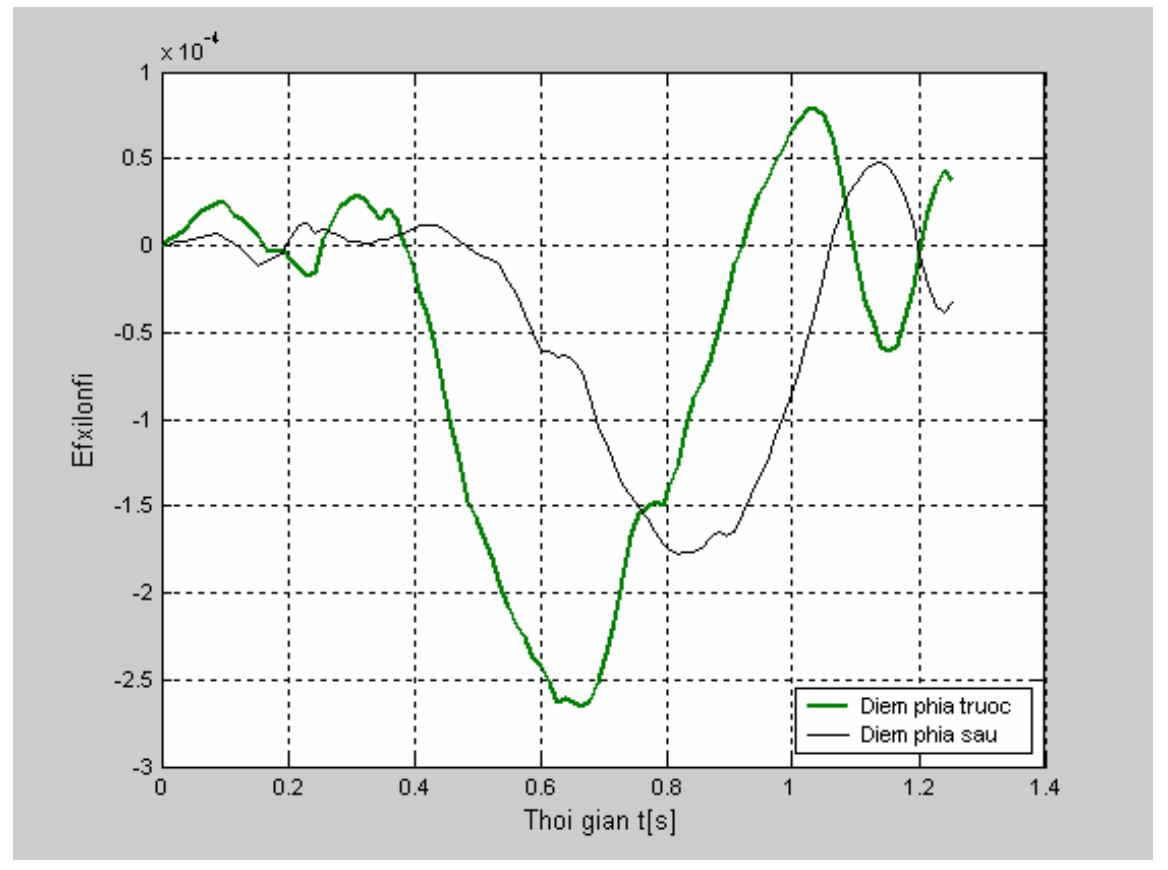

Hình 7: Biến dạng $\varepsilon_{\mathrm{s}}$ tại các điểm các điểm tính

Sử dụng bộ chương trình phần mềm đã lập, tiến hành tính toán, với: biên miền nghiên cứu $\mathrm{B}_{0}=3.5 \mathrm{~m}, \mathrm{H}_{0}=4 \mathrm{~m}, \mathrm{~L}_{0}=3 \mathrm{~m}$ (sai số $\varepsilon=0.5 \%$ ), thời gian tính $\mathrm{t}_{\mathrm{cal}}=8 \mathrm{~s}$, sai số chuyển vị $\varepsilon_{\mathrm{D}}=0.25 \%$. Kết quả cho ta biến dạng dài tỷ đối theo phương tiếp tuyến $(0 \varphi)$ và phương đường sinh (os) tại điểm giữa ( 2 điểm đối xứng nhau): điểm phía trước (gần mặt tác dụng tải) và phía sau (xa mặt tác dụng tải) của vỏ, tương ứng nút 128 và nút 164 của mô hình PTHH được thể hiện như trên hình 6 và 7 . Tóm tắt giá trị biến dạng lớn nhất của các điểm tính thể hiện như bảng 3 .

Bảng 3: Giá trị so sánh biến dạng lớn nhất (điểm phía trước và sau)

\begin{tabular}{|c|c|c|c|}
\hline \multicolumn{2}{|c|}{ Biến dạng $\varepsilon_{\varphi}\left(\times 10^{-4}\right)$} & \multicolumn{2}{c|}{ Biến dạng $\varepsilon_{\mathrm{s}}\left(\times 10^{-4}\right)$} \\
\hline Điểm trước & Điểm sau & Điểm trước & Điểm sau \\
\hline 2.6558 & 1.7765 & 1.2790 & 0.6012 \\
\hline
\end{tabular}

Nhận xét:

- Biến dạng tại các điểm tính (phía trước và phía sau) có sự chênh lệch về giá trị là khá lớn: biến dạng tại điểm tính phía trước lớn hơn tại điểm tính phía sau 33.1\% (đối với $\varepsilon_{\varphi}$ ) và $53.0 \%$ (đối với $\varepsilon_{\mathrm{s}}$ ). Các biến dạng này có quy luật phù hợp với quy luật cơ học.

- Thuật toán và chương trình tính toán đã lập có khả năng giải được các bài toán tương tác động lực học cũng như tương tác tĩnh học giữa kết cấu và nền san hô. 


\section{KẾT LUẬN}

Nội dung bài báo đã đạt được:

- Xây dựng mô hình, thuật toán PTHH và chương trình phần mềm trong môi trường Matlab giải bài toán tương tác giữa kết cấu dạng vỏ và nền san hô dưới tác dụng của tải trọng động.

- Tính toán trên ví dụ cụ thể là vỏ trụ kín nằm thẳng đứng trong nền san hô chịu tác dụng của tải trọng do nổ trong nền gây nên, kết quả tính phù hợp quy luật cơ học.

- Kết quả là tài liệu tham khảo và công cụ tính toán tương tác giữa kết cấu dạng vỏ và nền san hô chịu tải trọng tĩnh và động.

Công trình này hoàn thành dưới sự hỗ trợ của đề tài KC.09.07/06-10 và Chương trình KC.09/06-10

\section{TÀI LIỆU THAM KHẢO}

1. Nguyễn Thái Chung, 2006. Nền san hô và sự làm việc của cọc trong nền san hô, Luận án TSKT, Học viện KTQS.

2. Hoàng Xuân Lượng, 2004. Báo cáo tổng kết Đề tài KC.09.08, Trung tâm thông tin Khoa học kỹ thuật Quốc gia.

3. Bathe K.J., 1996. Finite element procedures, part one, two, Prentice Hall International Inc, Englewoob Cliff.

4. Bathe K.J., and Wilson E.L., 1978. Numerical Method in Finite Method Analyis Prentice, Hall of India Private Limited, New Delhi.

5. Clough R. and Penzien J., 1993. Dynamics of structures, Second edition, McGraw - Hill, Inc., ISBN 0-07-011394-7.

6. Goodman R.E., and Dubois J.J., 1972. Duplication of Dilatancy in Analysis of Jointed Rocks, Journal of Soil Mechanics and Foundations Div., ASCE, Vol. 98, No SM4, 399-422.

7. Pizhong Qiao, Jialai Wang, 2004. Mechanics and fracture of crack tip deformable bi-material interface, International Journal of Solids and Structures 41, 7423-7444.

8. Tzamtzis A.D., and Nath B., 1992. Application of a three-dimensional interface element to non-linear static and dynamic finite analysis of discontinuous systems, Engineering Systems Design and Analysis Conference, ASME, Vol.1, 219-222. 


\section{INTERACTION BETWEEN CYLINDRICAL SHELL AND CORAL FOUNDATION UNDER BLAST LOADING}

\section{NGUYEN THAI CHUNG}

Summary: In this study, the author present the calculating method for and some results studying interaction between cylindrical shell and coral foundation of Truong Sa archipelago under blast loading. The problem analysis with finite element method and using shell element (for shell structure), solid element (for coral foundation), 3D slip element (for layer between shell structure and coral foundation). The paper results can be used in design as reference for shell structures in the coral foundation under static and dynamic loading.

Ngày nhận bài: 03 - 02 - 2010

Ngưòi nhận xét: GS. TS. Hoàng Xuân Lượng 